\title{
Mesopic and dark-adapted two-color fundus-controlled perimetry in geographic atrophy secondary to age-related macular degeneration
}

\author{
Maximilian Pfau ${ }^{1,2}$, Philipp L. Müller ${ }^{1,3}$, Leon von der Emde ${ }^{1}$, Moritz Lindner ${ }^{1,4}$, Philipp T. \\ Möller $^{1,2}$, Monika Fleckenstein ${ }^{1,2}$, Frank G. Holz ${ }^{1,2}$, Steffen Schmitz-Valckenberg ${ }^{1,2}$ \\ 1. Department of Ophthalmology, University of Bonn, Ernst-Abbe-Str. 2, Bonn, Germany \\ 2. GRADE Reading Center, University of Bonn, Ernst-Abbe-Str. 2, Bonn, Germany \\ 3. Center for Rare Diseases, University of Bonn, Bonn, Germany \\ 4. The Nuffield Laboratory of Ophthalmology, Sleep and Circadian Neuroscience \\ Institute, Nuffield Department of Clinical Neurosciences, University of Oxford, Oxford, \\ United Kingdom
}

Running head: $\quad$ Mesopic and dark-adapted perimetry in GA

Number of words: $\quad 4,838$ (excluding title page, abstract, legends, and references)

Number of figures: $\quad 4$

Number of tables: 2

Supplemental Digital Content: 2

Correspondence:

Steffen Schmitz-Valckenberg, MD, FEBO

Department of Ophthalmology

University of Bonn

Ernst-Abbe-Str. 2

53127 Bonn

Germany

Tel.: $\quad$ +4922828716826

Fax: $\quad$ +49 22828711470

E-mail: steffen.schmitz-valckenberg@ukbbonn.de

Disclosure of funding:

The authors have no proprietary interest.

Funding/Support: This work was supported by the BONFOR Program of the Faculty of Medicine, University of Bonn [Grant No O-137.0022 and O-137.0025 to MP, Grant No O137.0023 to PLM] and by the German Research Foundation [Grant No 658/4-1 and 658/4-2 to MF, 2846/1-1 to ML and MU4279/1-1 to PM].

Financial Disclosures: CenterVue SpA, Padova, Italy has provided research material (SMAIA) for the conduct of this study. CenterVue had no role in the design or conduct of the experiments.

Acknowledgements:

The authors thank Joanna Czauderna and Ruth Hassenrik for patient coordination and data acquisition. 


\section{Key words}

microperimetry; dark-adapted two-color perimetry; scotopic sensitivity; macular disease; geographic atrophy

\section{Summary statement}

Mesopic and dark-adapted two-color perimetry may now be performed with fundus tracking facilitating sensitivity testing in patients with instable fixation. Patient-tailored perimetry grids were developed to allow for refined testing of the junctional zone in geographic atrophy. The results indicate that in proximity to the GA boundary, rod-dysfunction exceeds conedysfunction. 


\section{Abstract}

Purpose: To investigate retinal sensitivity in the junctional zone of geographic atrophy (GA) secondary to age-related macular degeneration using patient-tailored perimetry grids for mesopic and dark-adapted two-color fundus-controlled perimetry (FCP).

Methods: Twenty-five eyes with GA of 25 patients (prospective, natural-history study DSGA [Directional-Spread-in-Geographic-Atrophy; NCT02051998]) and 40 eyes of 40 normal subjects were included. Patient-tailored perimetry grids were generated using annotated fundus-autofluorescence data. Customized software positioned test-points along iso-hulls surrounding the GA-boundary at distances of $0.43^{\circ}, 0.86^{\circ}, 1.29^{\circ}, 2.15^{\circ}$ and $3.01^{\circ}$. The grids were used for duplicate mesopic and dark-adapted two-color (cyan and red) FCP. Ageadjusted reference-data were obtained through regression analysis of normative data followed by spatial interpolation.

Results: The mean sensitivity loss for mesopic testing decreased with the distance to GA ($10.3 \mathrm{~dB}\left[0.43^{\circ}\right],-8.2 \mathrm{~dB}\left[0.86^{\circ}\right],-7.1 \mathrm{~dB}\left[1.29^{\circ}\right],-6.8 \mathrm{~dB}\left[2.15^{\circ}\right]$ and $\left.-6.6 \mathrm{~dB}\left[3.01^{\circ}\right] ; \mathrm{P}<0.01\right)$. Dark-adapted cyan sensitivity-loss exceeded dark-adapted red sensitivity-loss for all iso-hulls ( -14.8 vs. $-11.7 \mathrm{~dB},-13.5$ vs. $-10.1 \mathrm{~dB},-12.8$ vs. $-9.1 \mathrm{~dB},-11.6$ vs. $-8.2 \mathrm{~dB},-10.7$ vs. $-8.0 \mathrm{~dB}$; $P<0.01)$

Conclusions: Patient-tailored FCP grids allowed for testing of retinal function in the junctional zone of GA with high spatial resolution. A distinct decrease in mesopic sensitivityloss between $0.43^{\circ}(125 \mu \mathrm{m})$ and $1.29^{\circ}(375 \mu \mathrm{m})$ was observed that leveled off at more distant test-points. In proximity to the GA boundary, the results indicate that rod- exceeded cone-dysfunction. 


\section{Introduction}

Geographic atrophy $(\mathrm{GA})$, the nonexudative late stage manifestation of age-related macular degeneration (AMD), leads to irreversible loss of visual function over time. ${ }^{1-6} \mathrm{GA}$ represents the predominant late form of AMD in the population 85 years of age or older. ${ }^{7,8}$ Histologically, GA is characterized by loss of retinal pigment epithelium (RPE) as well as a degeneration of the outer layers of the neurosensory retina and the choriocapillaris. ${ }^{3,4,9}$

The topographic manifestation of GA is variegated, yet not random. ${ }^{4,10-13}$ Typically, lesions first appear in the perifoveal macula commonly sparing the fovea. ${ }^{4,10-13}$ Best-corrected visual acuity (BCVA), the most frequently used functional endpoint in clinical trials in ophthalmology, therefore does not reflect GA progression adequately. ${ }^{12-16}$ Contrary, patients may also present with early foveal involvement resulting in stable BCVA measurements over time too, though, at lower levels..$^{6,14,17}$ Recent clinical trials have thus used fundus autofluorescence (FAF) imaging to quantify progression of GA areas over time. ${ }^{18-20} \mathrm{RPE}$ degeneration leads to the absence of the therein contained fluorophores resulting in areas of clearly-defined decreased FAF levels. ${ }^{18-20}$ However, outer retinal atrophy in the absence of RPE atrophy has been also described in the setting of AMD highlighting that visual function abnormalities may not always correlate with (detectable) structural findings in all image modalities. $^{21,22}$ It is therefore mandatory to develop and characterize novel functional clinical trial endpoints for GA secondary to AMD that allow for direct assessment of functional impairment and that are acceptable to regulatory authorities, health technology assessment bodies, and payers.

Fundus-controlled perimetry (FCP [also called microperimetry]) allows for spatially-resolved sensitivity testing even in the absence of stable fixation. ${ }^{23}$ While previous studies have used FCP to assess sensitivity loss in eyes with GA, the applicability of the previously used test protocols for clinical trials is limited. ${ }^{24-35}$ First, testing was performed in all studies using predefined FCP grids (e.g. 10-2), i.e. without considering the individual distribution of atrophic 
areas. Hence, the spatial resolution in junctional zone of GA, the main region of interest for longitudinal studies, was variable and often relatively low. While the foveo-papillary profile was shown to be useful as testing pattern in inherited monogenetic retinal diseases such as $A B C A 4$-associated retinopathy, ${ }^{36}$ it appears to be less promising in the setting of GA, since GA does not manifest necessarily along the horizontal meridian. ${ }^{11}$ Second, the retestvariability in proximity to the GA boundary, which is a prerequisite to differentiate measurement variability from clinically significant changes, has not been described. Third, the physiological hill of vision (i.e. normative data) has not been taken into account by the majority of previous investigators. As a result, studies investigating a potential increase of sensitivity with increasing distance to the GA boundary are conflicting. In this regard, recent work by Dennis and Astle (2016) on spatial interpolation of FCP data provided the essential framework to quantify sensitivity loss as compared to normative data for any test-point without any restriction to a fixed test pattern. ${ }^{37}$ Fourth, previously used mesopic FCP probes primarily cone-mediated vision. ${ }^{38}$ Given that rod-degeneration appears to exceed conedegeneration in $A M D,{ }^{39-42}$, efficient methods for quantifying rod-mediated vision are needed. The recently introduced S-MAIA (Scotopic Macular Integrity Assessment, CenterVue, Padova, Italy) device allows for dark-adapted (DA) two-color fundus-controlled perimetry (FCP), providing the opportunity to quantify rod-dysfunction even in patients with poor fixation. ${ }^{43-46}$

Here, we describe a novel approach to test function with high spatial resolution in the junctional zone of GA using patient-tailored perimetry grids, which were generated using annotated fundus autofluorescence data. Further, we provide retest-variability data that define the clinical significance of change in point-wise function. We outline a workflow that considers age-adjusted reference data and describe the retinal function in the junctional zone of GA including the distinction between mesopic as well as DA cyan and red sensitivity. 


\section{Methods}

\section{Patients}

Patients were recruited in the context of the Directional Spread in Geographic Atrophy (DSGA) study (NCT02051998). This non-interventional, prospective natural history study adhered to the tenets of the Declaration of Helsinki and was approved by the institutional review board (Institutional Review Board of the University of Bonn, approval ID: 197/12). Written informed consent was obtained from each participant after explanation of the study's nature and possible consequences of participation. The inclusion and exclusion criteria of the DSGA study have been reported previously. ${ }^{12}$ Briefly, patient had to be at least 55 years of age and exhibit uni- or multifocal GA, defined as any sharply demarcated round or ovalshaped lesion of at least $0.05 \mathrm{~mm}^{2}$ in area size and with clearly reduced FAF in the context of AMD-associated changes (i.e. large drusen and pigment abnormalities). Exclusion criteria included any signs of active or regressed exudation (e.g., bleeding, exudates, fibrous scar) as well as refractive errors $>5.00$ diopters of spherical equivalent or $>2.50$ diopters of astigmatisms in the study eye. Further exclusion criteria were the presence of other retinal diseases such as diabetic retinopathy and retinal dystrophy, as well as a history of laser photocoagulation or retinal surgery. The current sub-study included eyes from patients with unilateral or bilateral GA secondary to AMD that were motivated to undergo the extensive FCP examination. If both eyes met the inclusion criteria, the eye with less GA foci, followed by smaller total atrophic lesion size was included.

\section{Imaging}

Following pupil dilatation with $0.5 \%$ tropicamide and $2.5 \%$ phenylephrine, all patients underwent $30^{\circ} \times 30^{\circ}$ fundus autofluorescence imaging ( $\lambda$ excitation $488 \mathrm{~nm}, \lambda$ emission 500 $700 \mathrm{~nm}), 30^{\circ} \times 30^{\circ}$ infrared reflectance $(\lambda 815 \mathrm{~nm})$ imaging and $30^{\circ} \times 25^{\circ}$ SD-OCT imaging (121 B-scans, ART 25) using a Spectralis HRA+OCT2 (Heidelberg Engineering, Germany). Areas of GA were semi-automatically annotated using the RegionFinder software (Heidelberg Engineering, Germany) as previously described. ${ }^{18,19}$ 


\section{Patient-tailored perimetry grids}

Patient-tailored FCP grids were generated using custom-built software developed in the software environment MATLAB (MathWorks, United States). The software traced the boundaries of annotated GA lesions and placed test-points along „iso-hulls” surrounding the GA boundary at distances of $0.43^{\circ}\left(125 \mu \mathrm{m}\right.$ [in an emmetropic eye]), $0.86^{\circ}(250 \mu \mathrm{m}), 1.29^{\circ}$ $(375 \mu \mathrm{m}), 2.15^{\circ}(626 \mu \mathrm{m})$ and $3.01^{\circ}(876 \mu \mathrm{m})$, respectively (Figure 1). The distances represented multiples of the visual angle of a Goldmann III stimulus $\left(0.43^{\circ}\right)$. The first three equidistant test-points were placed considering previous histopathologic studies demonstrating a distinct progression of RPE dysmorphia between $500 \mu \mathrm{m}$ to $100 \mu \mathrm{m}$ from the GA boundary. ${ }^{4,9}$ Further, test-points (i.e. $-0.645^{\circ}$ ) were placed within the largest single lesion in each eye. The between test-point distance on each iso-hull was set to $1.51^{\circ}$ (437 $\mu \mathrm{m})$ aiming for an average of 60 test-points per eye equating to examination times of 10 minutes based on previous work. These grids were then saved as XML file and imported in the S-MAIA.

\section{Fundus-controlled perimetry}

A brief mesopic practice examination was performed in patients with no prior perimetry or FCP experience. Six different FCP exams were carried out in each patient, always using the identical patient-tailored grid: (1) duplicate mesopic (achromatic stimuli, 400-800 nm) FCP as well as (2) duplicate DA cyan (505) and (3) duplicated DA red (627 nm) testing. Prior to DA testing, patient underwent 35 min of dark adaption (light level $<0.1$ lux). The FCP grid position was adjusted slightly using the "Change grid position"-function of the S-MAIA if the preferred retinal locus (PRL) differed between the Spectralis and S-MAIA examination. For both mesopic and the DA cyan examinations, the follow-up mode was used for the second examination. For DA red testing, the follow-up mode was not used for the second examination as it was not available at the time of the study. All tests were performed with the preset 4-2 dB staircase strategy with a stimulus size of $0.43^{\circ}$ (Goldmann III). The exact test 
algorithm has been described previously in detail. ${ }^{43}$ The dynamic range of the S-MAIA device in this study was modified as compared to the prototype used in previous publications. ${ }^{43-46}$ The DA cyan and red stimuli covered now a dynamic range of $36 \mathrm{~dB}$ ranging from 0.00064 scotopic (scot.) $\mathrm{cd} / \mathrm{m}^{2}$ to $2.54 \mathrm{scot}$. $\mathrm{cd} / \mathrm{m}^{2}$. The former $0 \mathrm{~dB}$ and $20 \mathrm{~dB}$ stimuli of the S-MAIA prototype are equivalent to the $10 \mathrm{~dB}$ and $30 \mathrm{~dB}$ stimuli of the current S-MAIA version. ${ }^{43-46}$

\section{Reference data}

In analogy to Dennis and Astle (2016), age-adjusted reference data were obtained through regression analysis of normative data followed by spatial interpolation (see Supplement, Supplemental Digital Content 1, which describes the spatial interpolation of normative data). ${ }^{37}$ These normative data was based on duplicate mesopic, DA cyan and DA red FCP in 40 eyes (19 right eyes, 21 left eyes) of 40 subjects (mean age $58.0 \pm 17.4$ years [range 21.8 - 82.1 years], 13 females eyes). No signs of any ocular disease or history of intraocular surgery (except of cataract surgery) had to be present in normal eyes. The perimetry grid for all three types of FCP testing consisted of 5 concentric rings at $1^{\circ}, 3^{\circ}, 5^{\circ}, 7^{\circ}$ and $9^{\circ}$ with 12 stimulus locations evenly distributed (angular distance of $30^{\circ}$ to each other). Further, along the horizontal meridian there were additional stimuli placed at $0^{\circ}, 2^{\circ}, 4^{\circ}, 6^{\circ}, 8^{\circ}$ and $10^{\circ}$ resulting in a total of 71 stimulus locations.

\section{Data analysis}

For all analyses, the data of left eyes were flipped horizontally. The perimetry data of the SMAIA was registered to the SD-OCT data (subretinal en-face image with Bruch's membrane as boundary, mean intensity projection, slab thickness of $200 \mu \mathrm{m}$ ) using the moving least squares (non-linear) method (alpha 1.0, mesh resolution 32, affine transformation) as implemented in ImageJ (U.S. National Institutes of Health, Bethesda, Maryland, USA). The precise location of the foveal center point (outer nuclear layer in direct contact with the inner limiting membrane) was annotated in the SD-OCT data. Thus, the position of all test-points could be accurately defined by the distance to the A-scan of the foveal center point. These 
coordinates were then used to extract for all test-points reference data from the age-adjusted mean and 5 th percentile surfaces for all three types of testing.

\section{Statistical analyses}

Statistical analyses were performed using the software environment $R$ and the package nlme. Visual acuity measurements (Snellen fractions) were converted to the base-10 logarithm of the minimum angle of resolution (logMAR). Linear mixed-effects models were used to account for the hierarchical structure of the data (stimulus location nested in eye). Data from the $-0.645^{\circ}$ iso-hull within atrophy were excluded for all mixed-effects model analyses due to the highly skewed (non-normal) distribution of the results caused by floor effects. First, mixed-effects model analyses were used to examine the effect of test-pointspecific factors (i.e., distance to the GA border, retinal sensitivity) on the point-wise testretest variance. The point-wise coefficient of repeatability $(\mathrm{CoR})$, the value below which the absolute differences between two repeated measurements would lie with 0.95 probability, was calculated based on the within-subject retest-variance $\left(\mathrm{s}_{\mathrm{w}}{ }^{2}, \operatorname{CoR}= \pm 1.96 \times \sqrt{ }\left(2 \mathrm{~s}_{\mathrm{w}}{ }^{2}\right)\right)$. Second, the coefficient-of-determination $\left(R^{2}\right)$ for multiple linear regression was used to determine the between-subject variance in retest-variability explained by „patient reliability indices" (e.g. mean sensitivity, rate of false-positive responses, rate of wrong pressure events, fixation stability and age). The $R^{2}$ contribution for the individual „patient reliability index" was determined by averaging over orderings among regressors. The rate of falsepositive responses was defined as the rate of responses to a suprathreshold stimulus presented to the patient's blind spot (i.e. Heijl-Krakau method). The rate of wrong pressure events was defined as the number pressure events outside of the response window of the SMAIA divided by the examination time. ${ }^{47}$ The $95 \%$ bivariate contour ellipse area (BCEA) encompassing $95 \%$ of the fixation points was evaluated as measure of fixation stability. ${ }^{48}$ Third, the sensitivity in dependence of the iso-hull (i.e. distance to the boundary of GA) was determined using mixed-effects models. Hereby, the point-wise mean values of the first and second test were evaluated. Forth, the photoreceptor source was assessed based on the 
spectral sensitivity differences in DA testing. For the latter and the following analysis, data from test-points with an eccentricity of $\geq 2^{\circ}$ were included in consideration of the rod-free zone (diameter $\left.1.25^{\circ}\right) .{ }^{49}$ Last, we investigated the potential correlation of DA red and DA cyan sensitivity loss with mesopic sensitivity loss. 


\section{Results}

Design and feasibility of patient-tailored FCP grids in geographic atrophy

A total of 25 patients with GA secondary to AMD (age [mean \pm SD] $77.0 \pm 6.8$ years [range 64.1 - 89.7 years]) with a mean visual acuity of $0.53 \pm 0.42 \log$ MAR were included in the study (Table 1). Fourty normal eyes of 40 subjects were used to obtain normative sensitivity data through interpolation (see Supplement, Supplemental Digital Content 1). Based on the annotated imaging data, the developed software tool allowed for prompt generation of patient-tailored perimetry grids.

\section{Influence of test-point-specific factors on retest-reliability}

The point-wise CoR was $\pm 6.64 \mathrm{~dB}$ for mesopic testing, $\pm 5.78 \mathrm{~dB}$ for $\mathrm{DA}$ cyan testing and $\pm 7.24 \mathrm{~dB}$ for DA red testing. Mixed-effects model analyses were used to examine the effect of test-point-specific factors (i.e., distance to the GA border, retinal sensitivity) on the pointwise retest-variance. For mesopic testing, higher retinal sensitivity was associated with minimally less retest-variance ([slope estimate \pm standard error] $-0.26 \pm 0.08 \mathrm{~dB}^{2} / \mathrm{dB}$; $X 2(1)=10.288, P<0.01$ ). The specific iso-hull (i.e. the distance to the GA border) had no influence on the retest-variance $(X 2(4)=5.228, P=0.26)$. Similarly, in DA cyan testing, the retinal sensitivity had an effect on the retest-variance $\left(+0.28 \pm 0.07 \mathrm{~dB}^{2} / \mathrm{dB} ; \mathrm{x} 2(1)=14.334\right.$, $\mathrm{P}<0.001)$, while the iso-hull had no influence $(\mathrm{X} 2(4)=3.654, \mathrm{P}=0.45)$. Last, in DA red testing, both the test sensitivity $(X 2(1)=5.673, P=0.017)$ and distance to the GA border $(X 2(4)=19.29$, $\mathrm{P}<0.001)$ affected the retest-variance. A likelihood ratio test for DA red testing revealed that the model including only the distance to GA was superior to a model including both, the distance to GA and test sensitivity. A post-hoc test disclosed that the $2.15^{\circ}$ iso-hull $(-5.90 \pm$ $\left.1.40 \mathrm{~dB}^{2}\right)$ and $3.01^{\circ}$ iso-hull $\left(-4.51 \pm 1.38 \mathrm{~dB}^{2}\right)$ exhibited a lower $(\mathrm{P}<0.001$ and $\mathrm{P}<0.01)$ retest-variance as compared to the $0.43^{\circ}$ iso-hull. Summarizing, differences in retestvariance across all three types of testing and all iso-hulls were rather minor, which was also evident based on visual inspection (Figure 2). 


\section{Influence of patient-specific factors on retest-reliability}

To determine the between-subject variance in retest-variability explained by „patient reliability indices", the coefficient-of-determination $\left(R^{2}\right)$ for multiple linear regressions was used. The mean sensitivity, wrong pressure event rate, false-positive response rate, fixation stability, exam duration, average reaction time and patient age could only explain $45.34 \%$ (mesopic), $55.12 \%$ (DA cyan) and $32.96 \%$ (DA red) of the between-subject variance in retest-variability as shown in Table 2. Hereby, the mean sensitivity was overall the most important and consistent predictor of retest-variability for all three types of testing, with poor sensitivity resulting in higher retest-variability (Table 2 ).

\section{Retinal function in the junctional zone of GA}

Data from the $-0.645^{\circ}$ iso-hull within atrophy had to be excluded for the following analysis due to the highly skewed (non-normal) distribution of the results secondary to floor effects. Nevertheless, the sensitivity losses of these test-points within GA were markedly lower as compared to all test-points outside of atrophy (median [IQR] sensitivity loss of $-22.9 \mathrm{~dB}[-23.4$ $--19.5 \mathrm{~dB}]$ for mesopic, $-18.5 \mathrm{~dB}[-19.7--17.0 \mathrm{~dB}]$ for DA cyan and -20.8 [-21.8 - -17.4 dB] for DA red testing). Mixed-effect model analysis revealed that the distance to GA had a marked influence on the mesopic sensitivity $(X 2(4)=2050.66, P<0.001$; Figure 3$)$. A post-hoc analysis revealed, that there were differences $(P<0.001$ and $P<0.01)$ between $0.43^{\circ}$ (sensitivity loss of [mean \pm SE] $-10.68 \pm 1.05 \mathrm{~dB})$ and $0.86^{\circ}(-8.97 \pm 1.05 \mathrm{~dB}$ ) as well as between $0.86^{\circ}$ and $1.29^{\circ}(-7.83 \pm 1.05 \mathrm{~dB})$. However, there were no distinct differences $(P>0.05)$ between the mesopic sensitivity losses at $1.29^{\circ}, 2.15^{\circ}(-7.42 \pm 1.04 \mathrm{~dB})$ and $3.01^{\circ}$ $(-7.27 \pm 1.04 \mathrm{~dB})$. For DA cyan testing, the distance to GA had an influence on the sensitivity, too (Figure 3). The post-hoc comparison revealed marked differences $(P<0.01)$ in sensitivity losses between $0.43^{\circ}(-16.25 \pm 1.37 \mathrm{~dB})$ and $0.86^{\circ}(-14.95 \pm 1.37 \mathrm{~dB})$ and a distinct difference $(P<0.01)$ between $1.29^{\circ}(-14.08 \pm 1.36 \mathrm{~dB})$ and $2.15^{\circ}(-12.69 \pm 1.36 \mathrm{~dB})$. For $\mathrm{DA}$ red testing, distance to GA had a similar effect on sensitivity as for mesopic testing (Figure 3). The sensitivity losses varied distinctly $(P<0.001)$ between $0.43^{\circ}(-12.57 \pm 1.31 \mathrm{~dB})$ and 
$0.86^{\circ}(-11.06 \pm 1.31 \mathrm{~dB})$, between $(P<0.01) 0.86^{\circ}$ and $1.29^{\circ}(-10.05 \pm 1.31 \mathrm{~dB}$ and between $(P<0.05) 1.29^{\circ}$ and $2.15^{\circ}(-9.28 \pm 1.31 \mathrm{~dB})$. The sensitivity losses did not differ markedly between $(P>0.05) 2.15^{\circ}$ and $3.01^{\circ}(-9.10 \pm 1.31 \mathrm{~dB})$. In brief, a distinct increase in mesopic and DA red sensitivity that levels off for test-points more distant than $1.29^{\circ}$ or $2.15^{\circ}$ to the GA boundary was observable as well as more pronounced DA cyan sensitivity loss in close proximity to the GA boundary.

\section{Rod versus cone degeneration}

To analyze the degree of rod versus cone dysfunction, we classified all test-points (with an eccentricity of $\geq 2^{\circ}$ ) according to the spectral sensitivity difference in DA testing. Test-points with a cyan-red sensitivity difference within normal limits (i.e. value above $5^{\text {th }}$ percentile surface) were classified as rod-mediated implying that both stimuli were detected by rods. Test-points with a reduced cyan-red difference were classified as mixed-mediated implying detection by cones for the $627 \mathrm{~nm}$ stimulus and detection by rods for the $505 \mathrm{~nm}$ stimulus. These mixed-mediated test-points were sub-classified as mixed-mediated with DA red sensitivity within normal limits [a] and below normal limits [b]. Due to floor defects of the device, which prevented identification of isolated cone-mediation (cf. Limitations), test-points with a red sensitivity $<5 \mathrm{~dB}$ were classified as indeterminate. As show in Figure 3 , the percentage of rod-mediated test-points increased from (mean \pm SEM) $11.21 \pm 4.67 \%$ at a distance of $0.43^{\circ}$ to the GA boundary to $30.71 \pm 7.04 \%$ at a distance of $3.01^{\circ}$ to the GA boundary. Similarly, the percentage of cone-mediated test-points with DA red sensitivity within normal limits increased from $5.13 \pm 1.88 \%$ at $0.43^{\circ}$ to $12.69 \pm 3.30 \%$ at $3.01^{\circ}$. The percentage of test-points with indeterminate mediation decreased from $29.96 \pm 7.88 \%$ to $20.66 \pm 7.05 \%$. As shown in Figure 3, comparison of sensitivity losses estimated by DA FCP against mesopic FCP disclosed a strong linear correlation between DA red and mesopic sensitivity losses $\left(R^{2}=0.77\right)$. Hereby, the intercept of the linear mixed model was near zero $(0.58 \mathrm{~dB})$, and the slope was 0.89. Inspection of the second scatter plot in Figure 3 revealed a non-linear relationship between DA cyan and mesopic sensitivity losses. The non-linear 
relationship showed that DA cyan sensitivity loss occurred even in the absence of mesopic sensitivity loss. Contrary, mesopic sensitivity loss appeared to be always associated with DA cyan sensitivity loss, suggesting that DA cyan sensitivity loss (temporally) precedes mesopic sensitivity loss. 


\section{Discussion}

With this study, we introduce a novel and optimized approach to analyze functional impairment in GA secondary to AMD. Following the assessment of the individual configuration of atrophic lesions by FAF imaging, patient-tailored grids were generated and applied for mesopic and DA two-color FCP in order to specifically assess retinal function in the junctional zone of atrophy. Furthermore, the topographic variations of retinal sensitivity in normal eyes as well as the retest-variabilities - also with regards to the distance to atrophy, the degree of relative sensitivity loss and other patient-specific factors - were fully considered to define the clinical significance of change in point-wise function. This strategy allows for a more precise and detailed structure-function analysis in patients with GA secondary to AMD. Moreover, it may be used as a novel functional clinical trial endpoint in this so far untreatable and common cause for severe visual loss.

\section{Sensitivity in the junctional zone}

The gradual relative sensitivity loss towards the GA border, which was consistent among the three types of testing in this study, is consistent with both previous histopathological reports and findings from in-vivo imaging investigations ${ }^{2-4,9,50}$. The landmark study by Sarks and coworkers described the junctional zone of GA as a spatial sequence of degenerative changes towards the area of complete atrophy and pointed out that this zone may serve as a surrogate for degeneration over time..$^{3,4}$ These findings were later confirmed by in vivo SDOCT observations by our group in $2008 .^{50}$ Recently, Zanzottera and Ach et al. (2016) provided a detailed quantification demonstrating a distinct progression of RPE dysmorphia between $500 \mu \mathrm{m}$ to $100 \mu \mathrm{m}$ from the GA boundary that was associated with thickening of basal laminar deposits. ${ }^{9,51}$ These ex-vivo observations would be well in accordance with the FCP results of this in-vivo study, demonstrating - for example - that mesopic sensitivity progressively and distinctly increased between the iso-hull with a distance of $125 \mu \mathrm{m}\left(0.43^{\circ}\right)$, $250 \mu \mathrm{m}\left(0.86^{\circ}\right.$ iso-hull $)$ and then $375 \mu \mathrm{m}\left(1.29^{\circ}\right.$ iso-hull $)$ to the GA boundary. For more distant test-points, a rather uniform sensitivity loss was noted (Figure 3). 
Two additional conclusions may be drawn. First, the results confirm that FAF based quantification of GA in AMD (as accepted by the U.S. Food and Drug Administration as primary anatomical clinical endpoint in GA clinical trials) represents accurately the areal extent of deep scotomata. ${ }^{52}$ Contrary, it was shown for $A B C A 4$-associated retinopathy that deep scotomata may extend beyond the area of RPE atrophy highlighting the importance of limiting the use of structural endpoints to specific diseases. ${ }^{28}$ Second, the profound knowledge on the structural and functional lateral extent of the junctional zone may have an impact on the efficacy of therapeutical interventions that aim to slow or halt atrophy progression: Assuming that a "point-of-no-return" has been reached in the junctional zone, i.e. a stage of structural and functional damage beyond which progression of atrophy will occur not modifiable by therapeutic interventions, any anticipated difference for development of manifest atrophy between intervention and placebo would be limited to retinal areas that are more distant than approximately $375 \mu \mathrm{m}$ to the GA-boundary at baseline, while no effect on longitudinal changes in atrophic lesion size would be measurable in the area of the junctional zone (i.e. less than approximately $375 \mu \mathrm{m}$ to the GA boundary at baseline). Specifically, the delay between treatment initiation and measurable therapeutic effects would be at least approximately three years, considering the previously published rate of the lateral spread of GA (median [Q1-Q3] $106.90 \mu \mathrm{m} /$ year [55.44-161.70]). ${ }^{53}$ These considerations may potentially play a role in the failure of previous interventional clinical trials in GA patients in which the primary endpoint was not met during review periods of 12 to 36 months. ${ }^{54}$ 


\section{Rod versus cone-dysfunction in the junctional zone}

The comparison of the functional results between the three types of testing suggests both rod and cone photoreceptor involvement in the junctional zone. Previous psychophysical and histopathologic studies have pointed towards increased vulnerability of rod photoreceptors in AMD. ${ }^{39-42}$ The steeper decline and the smaller effect size of mesopic and DA red in relation to DA cyan sensitivity losses in this study would be in accordance with this current understanding of photoreceptor involvement in AMD. Yet, this is the first study - to the best of our knowledge - to describe specifically the degree of rod-dysfunction in the junctional zone of GA. In addition, our observation that DA cyan sensitivity loss was measurable in a large proportion of test-points in absence of mesopic sensitivity loss, whereas mesopic sensitivity loss appeared to be always associated with DA cyan sensitivity loss, implies that rod dysfunction temporally precedes cone retinal sensitivity impairment in the junctional zone of GA secondary to AMD. We have initiated and currently perform a longitudinal study with patient-tailored FCP analysis to test this hypothesis.

\section{Patient reliability indices}

FCP is supposedly not affected by instable fixation, blinks and saccades due to fundustracking. ${ }^{23,36}$ Regarding test-point-specific factors, the observation of no distinct (considering the effect sizes) relationship of the retest-variability to both the mean sensitivity and the distance to GA (i.e. iso-hull) - as consistently found for all three types of testing - would imply that functional changes over time along with the dynamic progression of both structure and function could be detected with similar variability and reliability. Importantly, „patient reliability indices" as global factors potentially affecting all test-points could only explain $33 \%$ to $55 \%$ of the variance in retest-variability among patients. Upcoming clinical trials (that aim for inclusion of patients with high retest-reliability) should therefore include and exclude patients rather based on the actual retest-reliability determined by a test and retest than based on „patient reliability indices”. The S-MAIA provides two measures of false-positive responses. The rate of false-positive responses to suprathreshold stimuli to the optic nerve 
head (i.e. Heijl-Krakau method), which was used as exclusion criterion in previous studies (e.g. with a cut-off of $>25 \%$ ), ${ }^{55}$ provided in our study only inconsistent information with regard to retest-variability while increasing the examination time. Wrong pressure events however (pressure events outside of the response window of the S-MAIA), which do not prolongate the examination time, were more informative with regard to retest-variability. Similar observations have been previously described in the setting of standard automated perimetry and glaucoma, suggesting that wrong pressure events could eliminate the necessity of falsepositive catch-trials in future studies. ${ }^{47}$

\section{Limitations}

The relative small number of subjects and the cross-sectional design represent limitations of this study. Furthermore, several additional limitations of current FCP testing methods have to be considered. The S-MAIA does not offer catch-trials to determine the false-negative response rate (i.e. random projection of suprathreshold stimuli to locations where sensitivity has already been measured). These may reduce the fraction of currently unexplained variance in retest-variability among patients. However, additional catch-trials would increase the examination times and potentially variability due to fatigue. Other forms of retestvariability such as intersession retest-variability were not examined in this study. Further, even with the extended dynamic range $(36 \mathrm{~dB})$ for DA testing, floor effects are observable. Considering the normative data for DA red testing (approx. $21 \mathrm{~dB}$ at an eccentricity of $5^{\circ}$ for a 77 year old subject), the spectral sensitivity difference between DA cyan and red testing that would indicate cone mediation for both stimuli (cyan-red difference of $-22 \mathrm{~dB}$ ) may not be detected with the device due to floor effects. This led to the classification of a subset of testpoints (putatively cone-mediated) as indeterminate. Longitudinal follow-up data will be needed to clearly demonstrate the superiority of patient-tailored FCP against BCVA in the setting of GA secondary to AMD. Last, eyes with a high number of foci may not be optimally probed using the here shown patient-tailored grids. The test times and a potential workflow 
for patient-tailored FCP in multicenter trials are provided (see Supplement, Supplemental Digital Content 2).

In summary, we described a novel approach to test retinal function with high spatial resolution in the junctional zone of GA evidencing a distinct increase in mesopic sensitivity that levels off for test-points more distant than approximately $375 \mu \mathrm{m}$ to the GA boundary as well as pronounced rod-dysfunction in close proximity to the GA boundary. As a prerequisite for patient-tailored FCP as functional clinical trial endpoint in GA, we (i.) provided retestvariability estimates that define the clinical significance of changes in function over time, (ii.) assessed the utility of „patient reliability indices” and (iii.) specified a workflow for multicenter studies with a central reading center. 


\section{References}

1. Gass JD. Drusen and disciform macular detachment and degeneration. Trans Am Ophthalmol Soc 1972; 70:409-436.

2. Green WR, Key SN. Senile macular degeneration: a histopathologic study. Trans Am Ophthalmol Soc 1977; 75:180-254.

3. Sarks SH. Ageing and degeneration in the macular region: a clinico-pathological study. Br J Ophthalmol 1976; 60:324-341.

4. Sarks JP, Sarks SH, Killingsworth MC. Evolution of geographic atrophy of the retinal pigment epithelium. Eye (Lond) 1988; 2:552-577.

5. Lim LS, Mitchell P, Seddon JM, Holz FG, Wong TY. Age-related macular degeneration. Lancet 2012; 379:1728-1738.

6. Schmitz-Valckenberg S, Nadal J, Fimmers R, et al. Modeling Visual Acuity in Geographic Atrophy Secondary to Age-Related Macular Degeneration. Ophthalmologica 2016; 235:215-224.

7. Klein R, Klein BE, Franke $T$. The relationship of cardiovascular disease and its risk factors to age-related maculopathy. The Beaver Dam Eye Study. Ophthalmology $1993 ; 100: 406-414$.

8. Klein R, Klein BEK, Knudtson MD, Meuer SM, Swift M, Gangnon RE. Fifteen-Year Cumulative Incidence of Age-Related Macular Degeneration. Ophthalmology 2007; 114:253-262.

9. Zanzottera EC, Ach T, Huisingh C, Messinger JD, Freund KB, Curcio CA. Visualizing Retinal Pigment Epithelium Phenotypes in the Transition To Atrophy in Neovascular Age-Related Macular Degeneration. Retina 2016; 36:S26-S39.

10. Sunness JS. The natural history of geographic atrophy, the advanced atrophic form of age-related macular degeneration. Mol Vis 1999; 5:25-30. 
11. Mauschitz MM, Fonseca S, Chang P, et al. Topography of geographic atrophy in agerelated macular degeneration. Invest Ophthalmol Vis Sci 2012; 53:4932-4939.

12. Lindner M, Böker A, Mauschitz MM, et al. Directional Kinetics of Geographic Atrophy Progression in Age-Related Macular Degeneration with Foveal Sparing. Ophthalmology 2015; 122:1356-1365.

13. Lindner M, Nadal J, Mauschitz MM, et al. Combined Fundus Autofluorescence and Near Infrared Reflectance as Prognostic Biomarkers for Visual Acuity in FovealSparing Geographic Atrophy. Invest Ophthalmol Vis Sci 2017; 58:BIO61-BIO67.

14. Sunness JS, Gonzalez-Baron J, Applegate CA, et al. Enlargement of atrophy and visual acuity loss in the geographic atrophy form of age-related macular degeneration. Ophthalmology 1999; 106:1768-1779.

15. Sunness JS. Reading newsprint but not headlines: pitfalls in measuring visual acuity and color vision in patients with bullseye maculopathy and other macular scotomas. Retin Cases Brief Rep 2008; 2:83-84.

16. Sayegh RG, Sacu S, Dunavolgyi R, et al. Geographic Atrophy and Foveal-Sparing Changes Related to Visual Acuity in Patients With Dry Age-Related Macular Degeneration Over Time. Am J Ophthalmol 2017; 179:118-128.

17. Sunness JS, Margalit E, Srikumaran D, et al. The Long-term Natural History of Geographic Atrophy from Age-Related Macular Degeneration. Enlargement of Atrophy and Implications for Interventional Clinical Trials. Ophthalmology 2007; 114:271-277.

18. Schmitz-Valckenberg S, Brinkmann CK, Alten F, et al. Semiautomated image processing method for identification and quantification of geographic atrophy in agerelated macular degeneration. Invest Ophthalmol Vis Sci 2011; 52:7640-7646.

19. Pfau M, Goerdt L, Schmitz-Valckenberg S, et al. Green-Light Autofluorescence Versus Combined Blue-Light Autofluorescence and Near-Infrared Reflectance Imaging in 
Geographic Atrophy Secondary to Age-Related Macular Degeneration. Invest Ophthalmol Vis Sci 2017; 58:BIO121-BIO130.

20. Holz FG, Sadda SR, Staurenghi G, et al. Imaging Protocols in Clinical Studies in Advanced Age-Related Macular Degeneration: Recommendations from Classification of Atrophy Consensus Meetings. Ophthalmology 2017; 124:464-478.

21. Spaide RF. Outer Retinal Atrophy After Regression of Subretinal Drusenoid Deposits As a Newly Recognized Form of Late Age-Related Macular Degeneration. Retina 2013; 33:1800-1808.

22. Sadda SR, Guymer R, Holz FG, et al. Consensus Definition for Atrophy Associated with Age-Related Macular Degeneration on OCT: Classification of Atrophy Report 3. Ophthalmology November 2017.

23. Rohrschneider K, Bültmann S, Springer C. Use of fundus perimetry (microperimetry) to quantify macular sensitivity. Prog Retin Eye Res 2008; 27:536-548.

24. Sunness J, Johnson M, Massof R. Retinal sensitivity over drusen and nondrusen areas. A study using fundus perimetry. Arch Ophthalmol 1988; 106:1081-1084.

25. Schmitz-Valckenberg S, Bultmann S, Dreyhaupt J, Bindewald A, Holz FG, Rohrschneider K. Fundus Autofluorescence and Fundus Perimetry in the Junctional Zone of Geographic Atrophy in Patients with Age-Related Macular Degeneration. Invest Ophthalmol Vis Sci 2004; 45:4470-4476.

26. Takahashi A, Ooto S, Yamashiro K, et al. Photoreceptor Damage and Reduction of Retinal Sensitivity Surrounding Geographic Atrophy in Age-Related Macular Degeneration. Am J Ophthalmol 2016; 168:260-268.

27. Hariri AH, Tepelus TC, Akil H, Nittala MG, Sadda SR. Retinal Sensitivity at the Junctional Zone of Eyes With Geographic Atrophy Due to Age-Related Macular Degeneration. Am J Ophthalmol 2016; 168:122-128. 
28. Sunness JS. What you see is not always what you get in atrophic macular disease. Retin Cases Brief Rep 2008; 2:205-208.

29. Hartmann KI, Bartsch DU, Cheng L, et al. Scanning laser ophthalmoscope imaging stabilized microperimetry in dry age-related macular degeneration. Retina 2011; $31: 1323-1331$.

30. Meleth $A D$, Mettu $P$, Agrón E, et al. Changes in retinal sensitivity in geographic atrophy progression as measured by microperimetry. Invest Ophthalmol Vis Sci 2011; 52:1119-1126.

31. Chen FK, Patel PJ, Webster AR, Coffey PJ, Tufail A, Da Cruz L. Nidek MP1 is able to detect subtle decline in function in inherited and age-related atrophic macular disease with stable visual acuity. Retina 2011; 31:371-379.

32. Landa G, Su E, Garcia PMT, Seiple WH, Rosen RB. Inner segment-outer segment junctional layer integrity and corresponding retinal sensitivity in dry and wet forms of age-related macular degeneration. Retina 2011; 31:364-370.

33. Querques L, Querques G, Forte R, Souied EH. Microperimetric correlations of autofluorescence and optical coherence tomography imaging in dry age-related macular degeneration. Am J Ophthalmol 2012; 153:1110-1115.

34. Pilotto E, Benetti E, Convento E, et al. Microperimetry, fundus autofluorescence, and retinal layer changes in progressing geographic atrophy. Can J Ophthalmol 2013; 48:386-393.

35. Sayegh R, Kiss C, Simader C, et al. A systematic correlation of morphology and function using spectral domain optical coherence tomography and microperimetry in patients with geographic atrophy. Br J Ophthalmol 2014; 98:1050-1055.

36. Cideciyan A V., Swider M, Aleman TS, et al. Macular function in macular degenerations: repeatability of microperimetry as a potential outcome measure for 
ABCA4-associated retinopathy trials. Invest Ophthalmol Vis Sci 2012; 53:841-852.

37. Denniss J, Astle AT. Spatial interpolation enables normative data comparison in gazecontingent microperimetry. Invest Ophthalmol Vis Sci 2016; 57:5449-5456.

38. Simunovic MP, Moore AT, MacLaren RE. Selective Automated Perimetry Under Photopic, Mesopic, and Scotopic Conditions: Detection Mechanisms and Testing Strategies. Transl Vis Sci Technol 2016; 5:10.

39. Curcio CA, Allen KA. Aging of the Human Photoreceptor Mosaic: Evidence for Selective Vulnerability of Rods in Central Retina. Invest Ophthalmol Vis Sci 1993; 34:3278-3296.

40. Owsley C, Jackson GR, Cideciyan A V., et al. Psychophysical evidence for rod vulnerability in age-related macular degeneration. Invest Ophthalmol Vis Sci 2000; $41: 267-273$.

41. Jackson GR, Owsley C, Curcio CA. Photoreceptor degeneration and dysfunction in aging and age-related maculopathy. Ageing Res Rev 2002; 1:381-396.

42. Owsley C, McGwin G, Clark ME, et al. Delayed Rod-Mediated Dark Adaptation Is a Functional Biomarker for Incident Early Age-Related Macular Degeneration. Ophthalmology 2016; 123:344-351.

43. Pfau M, Lindner M, Fleckenstein M, et al. Test-Retest Reliability of Scotopic and Mesopic Fundus-Controlled Perimetry Using a Modified MAIA (Macular Integrity Assessment) in Normal Eyes. Ophthalmologica 2017; 237:42-54.

44. Pfau M, Lindner M, Muller PL, et al. Effective Dynamic Range and Retest Reliability of Dark-Adapted Two-Color Fundus-Controlled Perimetry in Patients With Macular Diseases. Invest Ophthalmol Vis Sci 2017; 58:BIO158-BIO167.

45. Steinberg JS, Sassmannshausen M, Pfau M, et al. Evaluation of Two Systems for Fundus-Controlled Scotopic and Mesopic Perimetry in Eye with Age-Related Macular 
Degeneration. Transl Vis Sci Technol 2017; 6:7.

46. Pfau M, Lindner M, Steinberg JS, et al. Visual field indices and patterns of visual field deficits in mesopic and dark-adapted two-colour fundus-controlled perimetry in macular diseases. Br J Ophthalmol November 2017.

47. Olsson J, Bengtsson B, Heijl A, Rootzen H. An improved method to estimate frequency of false positive answers in computerized perimetry. Acta Ophthalmol Scand 1997; 75:181-183.

48. Steinman RM. Effect of Target Size, Luminance, and Color on Monocular Fixation. J Opt Soc Am 1965; 55:1158-1164.

49. Curcio C, Sloan K, Kalina R, Hendrickson A. Human photoreceptor topography. J Comp Neurol 1990; 4:497-523.

50. Fleckenstein M, Issa PC, Helb H-MM, et al. High-resolution spectral domain-OCT imaging in geographic atrophy associated with age-related macular degeneration. Invest Ophthalmol Vis Sci 2008; 49:4137-4144.

51. Rudolf M, Vogt SD, Curcio CA, et al. Histologic basis of variations in retinal pigment epithelium autofluorescence in eyes with geographic atrophy. Ophthalmology 2013; 120:821-828.

52. Csaky KG, Richman EA, Ferris FL. Report from the NEI/FDA ophthalmic clinical trial design and endpoints symposium. Invest Ophthalmol Vis Sci 2008; 49:479-489.

53. Fleckenstein M, Schmitz-Valckenberg S, Adrion C, et al. Tracking progression with spectral-domain optical coherence tomography in geographic atrophy caused by agerelated macular degeneration. Invest Ophthalmol Vis Sci 2010; 51:3846-3852.

54. Jaffe GJ, Schmitz-Valckenberg S, Boyer D, et al. Randomized Trial to Evaluate Tandospirone in Geographic Atrophy Secondary to Age-Related Macular Degeneration: The GATE Study. Am J Ophthalmol 2015; 160:1226-1234. 
55. Wu Z, Ayton LN, Guymer RH, Luu CD. Intrasession Test - Retest Variability of Microperimetry in Age-related macular degeneration. Assoc Res Vis Ophthalmol 2013; 54:7378-7385. 


\section{Figure captions}

\section{Figure 1. Patient-tailored perimetry}

Patient-tailored perimetry grids were generated using annotated fundus autofluorescence (FAF) and infrared-reflectance (IR) data $\left(30^{\circ} \times 30^{\circ}\right.$, Spectralis OCT2, Heidelberg Engineering, Germany). A customized software positioned test-points along iso-hulls surrounding the GA boundary at distances of $0.43^{\circ}\left(125 \mu \mathrm{m}\right.$ [in an emmetropic eye]), $0.86^{\circ}(250 \mu \mathrm{m}), 1.29^{\circ}(375$ $\mu \mathrm{m}), 2.15^{\circ}(626 \mu \mathrm{m})$ and $3.01^{\circ}(876 \mu \mathrm{m})$ to the GA boundary as outlined (Top right). The between test-point distance on each iso-hull was $1.51^{\circ}(437 \mu \mathrm{m})$. An exemplary grid for a given patient that was then used for duplicate mesopic (background $1.27 \mathrm{~cd} / \mathrm{m} 2$, achromatic stimuli) as well as dark-adapted two-color (cyan [505 nm] and red [627 nm] stimuli) FCP with a 4-2 staircase strategy (S-MAIA, CenterVue, Italy) is shown (Bottom left). The coordinates of each test-point in relation to the foveal center point (identified in spectral-domain optical coherence tomography) were used to extract precise reference data for each test-point from a database of spatially-interpolated age-adjusted normative data (Bottom right). This was done separately for all three types of testing for the age-adjusted mean and 5th percentile surfaces.

\section{Figure 2. Retest-variability}

The left column shows the point-wise sensitivity (PWS) test-retest differences in dependence of the distance to the GA for mesopic, dark-adapted cyan and dark-adapted red testing. The right column shows the PWS test-retest differences in dependence of the point-wise mean sensitivity (i.e. Bland-Altman plots) for mesopic, dark-adapted cyan and dark-adapted red testing. For all three types of testing, the retest-variability appears visually not to depend markedly on the distance to GA or test-point sensitivity. For mesopic and dark-adapted red testing a slight increased retest-variability for test-points with a mean sensitivity of 5 to $10 \mathrm{~dB}$ is observable. The solid line represents the mean test-retest difference and the dashed lines 
the $95 \%$ confidence intervals. To reduce overplotting, all points were plotted semitransparent in all plots and jittered along the x-axis in the left column.

\section{Figure 3. Sensitvity in the junctional zone of GA}

The upper panel shows the mean sensitivity loss of all patients for each iso-hull for each of the three types of testing. The results of the post-hoc tests for the mixed-effects models were indicated using asterisks ( ${ }^{* \star *}$ for $\mathrm{P}<0.001,{ }^{* *}$ for $\mathrm{P}<0.01$, * for $\mathrm{P}<0.05$ and n.s. for $\mathrm{P}>0.05$ ). Colored LOESS curves represent estimates of the conditional mean. For mesopic funduscontrolled perimetry (FCP), a distinct increase in sensitivity was observed between the $0.43^{\circ}$, $0.86^{\circ}$ and $1.29^{\circ}$ iso-hulls. Between $1.29^{\circ}$ and $3.01^{\circ}$ the sensitivity remained similar. Darkadapted (DA) red FCP yielded similar results. In DA cyan FCP, a slower increase in sensitivity was observable that did not appear to level-off within $3.01^{\circ}$ to the geographic atrophy boundary. The Bottom left panel shows the percentage of test-points (eccentricity $\geq$ $2^{\circ}$ ) categorized as rod-mediated (cyan-red sensitivity difference wnl), mixed-mediated (cyanred sensitivity difference reduced with DA red sensitivity wnl [a] or reduced [b]) and indeterminate-mediation (floor of the dynamic range of the device reached). The Bottom right panel shows the sensitivity losses for all test-points (eccentricity $\geq 2^{\circ}$ ). While a strong linear correlation between DA red sensitivity losses and mesopic sensitivity losses was observable, DA cyan sensitivity losses and mesopic sensitivity losses exhibited no linear relationship. DA cyan sensitivity loss occurred even in presence of normative mesopic function. Contrary, mesopic sensitivity loss appeared to be always associated with DA cyan sensitivity loss.

\section{Figure 4. Exemplary patients}

The left columns shows the results of the dark-adapted (DA) fundus-controlled perimetry (FCP) testing for two exemplary patients. The test-points were categorized according to the spectral sensitivity differences as rod-mediated (cyan-red sensitvity difference wnl), mixed- 
mediated (cyan-red sensitivity difference reduced with DA red sensitivity wnl [a] or reduced [b]) and indeterminate-mediation (floor of the dynamic range of the device reached). For graphical representation, the results were spatially interpolated using inverse distance weighting. The right columns show the corresponding fundus autofluorescence (FAF) images. The dashed circles surround the fovea with a radius of $2^{\circ}$ encompassing the rod-free zone (diameter of $1.25^{\circ}$ ). These test-points were excluded from the summary analyses in Figure 3. For patient 1, an obvious correlation between rod-dysfunction and irregularity of the FAF signal is visible. The temporal junctional zone exhibited mostly rod mediation and regular FAF signal. The nasal margin exhibit irregular FAF signal associated with roddysfunction. Similarly in patient 2, two spots of nascent atrophy (indicated by the arrow heads) were clearly associated with rod-dysfunction. 


\section{List of Supplemental Digital Content}

Supplemental Digital Content 1. Spatial interpolation of normative data. Pdf

Supplemental Digital Content 2. Test time and workflow for clinical trials. pdf 